\section{ASPP bites}

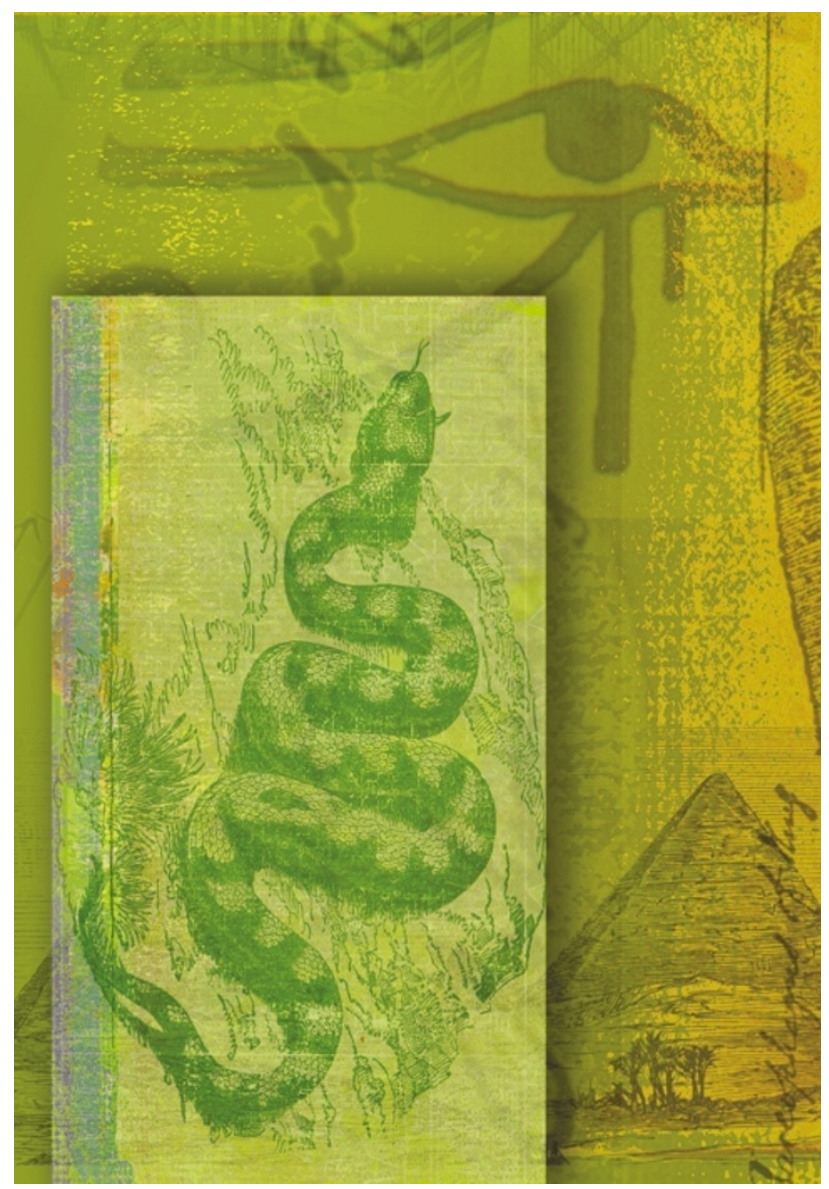

When stress signals activate $\mathrm{p} 53$, the cell has a choice between pausing the cell cycle and repairing any DNA damage, or - if the damage is too great - sentencing the cell to death by apoptosis. Yardena Samuels-Lev and colleagues in Xin Lu's lab now describe a family of proteins, the ASPPs (for apoptosis-stimulating protein of p53), that might influence this decision.

The ASPP family was found in a database search for homologues of a previously identified $\mathrm{p} 53$ binding protein, 53BP2. The authors discovered two sequences: one, which they have named ASPP2, is a long isoform of 53BP2; the other, ASPP1, is a novel protein. The residues known to interact with p53 from the previously solved crystal structure of the p53-53BP2 complex are conserved in both ASPPs, and immunoprecipitations confirmed that both interact with p53.

Does binding to ASPPs alter p53 function? Cotransfection of ASPP genes and TP53 into a TP53 ${ }^{-/-}$cell line caused a marked increase in the proportion of cells that underwent apoptosis compared with cells transfected with TP53 alone. 53BP2 also interacts with the anti-apoptotic protein BCL2, so could the proapoptotic effect of ASPP be due to an inhibitory effect on BCL2? Apparently not, because ASPP had no effect on apoptosis induced by E2F1 or BAX expression.
Furthermore, antisense oligonucleotides that block the production of ASPP1 and ASPP2 had no effect on BAX-mediated apoptosis.

So, how do ASPPs aid in this cellular euthanasia? Chromatin immunoprecipitation revealed that $A S P P 2$ expression caused an eightfold increase in the amount of p53 bound to the $B A X$ promoter, but it had no effect on the amount of $\mathrm{p} 53$ bound to the WAF1 promoter - the gene through which p53 induces cell-cycle arrest. ASPPs had even more dramatic effects on p53-mediated transactivation of the pro-apoptotic genes $B A X$ and PIG3, increasing their expression 20-30-fold, but had no effect on transactivation of p53 target genes with other functions, such as MDM2, cyclin G and WAF1.

The pro-apoptotic effects of ASPPs required their amino termini, indicating that 53BP2 - the N-terminally truncated form of ASPP2 - might be a dominant-negative mutant of ASPP2. In support of this, expression of $53 \mathrm{BP} 2$ reduced the ability of $\mathrm{p} 53$ to cause apoptosis. This raises the possibility that binding of ASPP's N terminus to another protein is involved in boosting p53's ability to transactivate pro-apoptotic target genes.

The discovery of ASPPs might also clear up a mystery concerning why two oncogenic p53 mutations, 181L and $181 \mathrm{C}$, can transactivate WAF1 but are very inefficient at stimulating
APOPTOSIS

\section{Escape artists}

Although cytotoxic T lymphocytes (CTLs) usually form an effective barrier against cancer, tumours have developed a number of mechanisms to escape the cellular immune response. In the 25 September issue of Proceedings of the National Academy of Sciences, Medema et al. report a new mechanism by which tumours evade T-cellmediated cytolysis.

$\mathrm{T}$ cells induce apoptosis in target cells through two mechanisms - death-receptor activation and perforin/granzyme $\mathrm{B}(\mathrm{GrB})$ induced exocytosis. GrB is secreted by CTLs and enters the target cell through receptormediated endocytosis. Once inside the cell, $\mathrm{GrB}$ is released from the endosome into the cytoplasm, where it cleaves substrates such as
BID and caspases, leading to apoptosis. The serine protease inhibitor (serpin) PI9 irreversibly inhibits GrB and prevents CTLmediated killing. This serpin has been shown to be expressed in lymphoid tissues and $\mathrm{T}$ cells, protecting CTLs from destruction by their own GrB. Medema et al. found that a variety of human tumours, including melanoma, breast, cervical and colon carcinoma, also express $P I 9$, whereas a number of mouse tumours express Spi6, the mouse homologue. Using an in vitro cytotoxicity assay, they showed that Spi6expressing mouse tumours are resistant to CTL-induced apoptosis, whereas Spi6negative tumour cell lines are not. The authors transfected the gene that encodes Spi6 into tumour cell lines that do not normally express the protein but are resistant to death-receptor-mediated apoptosis. These cells managed to evade CTLmediated lysis, whereas nontransfected cells did not. But does Spi6 confer a survival advantage in vivo? The authors co-injected fluorescently labelled Spi6-expressing tumour cells and nonexpressing tumour cells into mice, and observed that the transfected cells had a distinct survival advantage.

Several mechanisms have been reported by which tumour cells escape the death-receptormediated apoptotic pathway. Medema $\mathrm{et}$ al. offer the first demonstration of a mechanism by which tumours escape the perforin/GrB pathway. Cancer cells develop a number of techniques to interfere with the apoptotic signalling cascade; these must be identified before CTL-mediated immunotherapy can be further developed.

\section{Kristine Novak}

(2) References and links

ORIGINAL RESEARCH PAPER Medema, J. P. et al. Blockade of the granzyme $B /$ perforin pathway through overexpression of the serine protease inhibitor PI-9/SPI-6 constitutes a mechanism for immune escape by tumors. Proc. Natt Acad. Sci. USA 98, 11515-11520 (2001) 
apoptosis. These mutants coimmunoprecipitated poorly with ASPP1, although they seemed to interact well with ASPP2.

Might ASPP inactivation offer a selective advantage to tumours that have wild-type TP53? In a panel of 58 breast tumours with matched normal tissue, most of the TP53 wild-type tumours had reduced expression of either ASPP1 or (less commonly) ASPP2, whereas most of those with mutant TP53 had normal ASPP expression levels.

Cleopatra had an accomplice the asp - to help her die, and now, it seems, $\mathrm{p} 53$ also uses an ASPP to nudge cells towards death. The discovery of this family explains why the promoters of many of p53's target genes, particularly the pro-apoptotic ones, have weak p53 binding sites. By modulating p53's ability to bind at these promoters, the cell can control the decision to live or die. The precise mechanism by which ASPPs boost p53's killing power, and the tantalizing possibility of sensitizing tumours to chemotherapy or radiotherapy by reactivating $A S P P$ expression, are exciting avenues for future research.

Cath Brooksbank

\section{(1) References and links} ORIGINAL RESEARCH PAPER Samuels-Lev, Y. et al. ASPP proteins specifically stimulate the apoptotic functions of p53. Mol. Cell 8, 781-794 (2001)

\section{WEBSITES}

The p53 database: $\mathrm{http}: / /$ www.iarc.fr/p53/

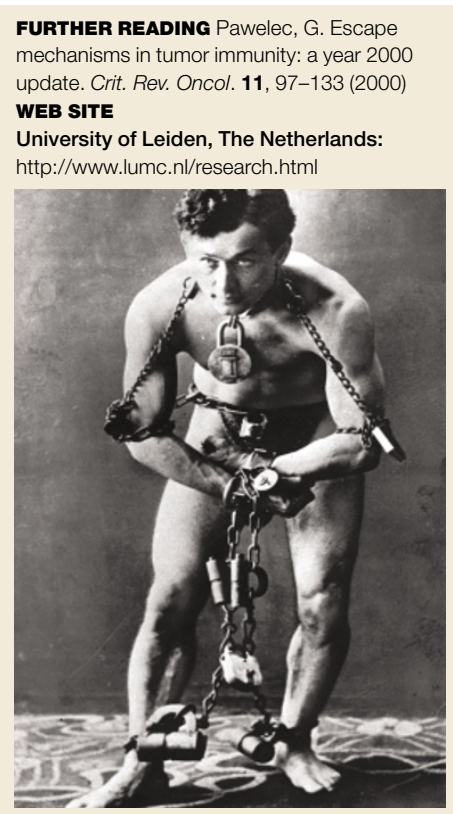

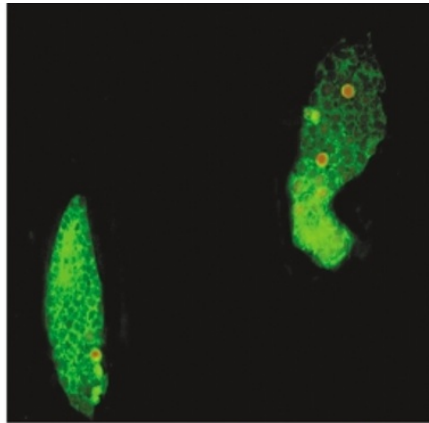
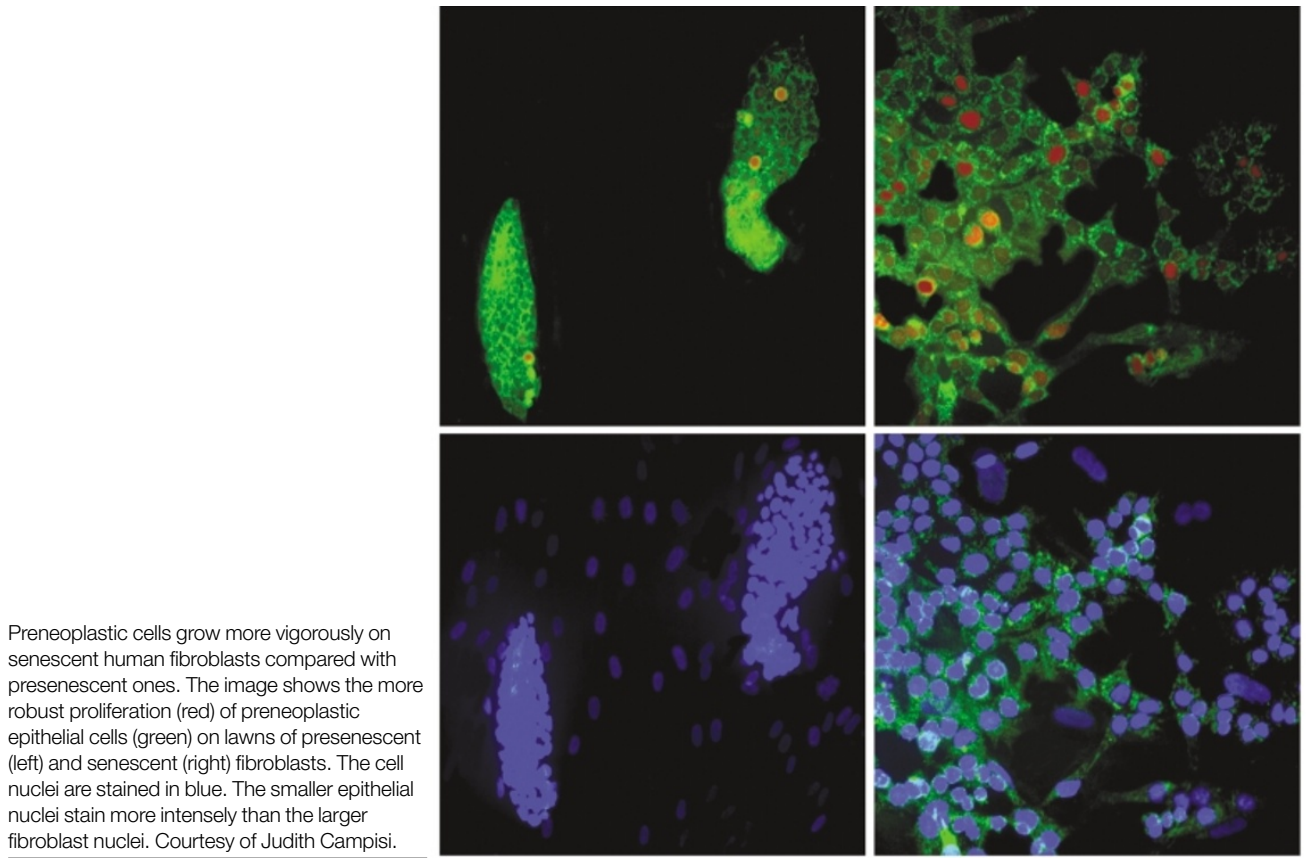

\section{SENESCENCE}

\section{The worm that turns}

It has long been known that the incidence of cancer increases as we age owing to the accumulation of mutations. But now Judith Campisi and colleagues, reporting in the 9 October issue of Proceedings of the National Academy of Sciences, show that another mechanism contributes to tumorigenesis as we grow old. Cellular senescence, which protects us from cancer when we are young (oncogenic stimuli can induce cellular senescence), slowly turns against us to promote tumour growth.

Cellular senescence causes remarkable changes in gene expression, and senescent fibroblasts secrete proteins - such as growth factors and enzymes - that can alter the microenvironment and could affect the growth of the epithelial cells that surround them. To investigate this, the authors incubated normal, preneoplastic and neoplastic epithelial cells with presenescent and senescent fibroblasts. Senescent fibroblasts could stimulate the growth of neoplastic and preneoplastic cells, but not of normal epithelial cells. The growth difference between cells incubated with senescent and presenescent cells was seen within just 4 days (see picture).

So, how do senescent cells stimulate epithelial cell growth? The two possibilities were through factors secreted by the senescent fibroblasts or through direct cell-cell interaction. To test this, the authors grew preneoplastic epithelial cells and senescent fibroblasts in chambers that were separated by a membrane, which prevented cell contact but allowed diffusion of soluble factors. A 2-3-fold increase in cell growth was seen with the senescent cells compared with the presenescent cells, indicating that soluble factors secreted from senescent cells can stimulate cell growth. But are secreted matrix proteins also involved? Senescent and presenescent fibroblasts were grown on culture dishes where they deposited extracellular matrix proteins. The fibroblasts were removed and preneoplastic cells were plated on top. Again, the matrix deposited by the senescent cells stimulated 3-4-fold more growth than the presenescent cells. The authors calculated that secreted factors account for at least $50 \%$ of the growth stimulation by senescent cells, but that cell-cell interaction is also involved.

So, if senescent cells can stimulate growth of preneoplastic cells, might they be able to induce tumorigenesis in vivo? Campisi and colleagues injected epithelial cells, either alone or with senescent fibroblasts, into immunocompromised mice. Injection of preneoplastic human epidermal keratinocytes (HaCATs) alone did not induce tumour formation by 40 days, but $7 / 15$ mice that were also injected with senescent cells had developed tumours. MDA231 cells, a human breast cancer cell line, were also injected into mice either alone or with senescent fibroblasts. In this case, small tumours had formed by 45 days in 2/5 mice injected with MDA231 cells, but when combined with senescent cells, more mice (4/5) developed tumours, and the tumours were significantly larger. So, senescent fibroblasts can stimulate tumorigenesis in mice.

Why should a process that evolved to protect us have such dire consequences later in life? The authors suggest that selection for the process has unforseen and unselected effects in aged organisms. Deleterious mutations accumulate in cells as they age, so the probability that mutant cells will be in close proximity to senescing cells increases, thereby reinforcing the tumorigenic process. Cancer cells have therefore found yet another way of coming out on top.

Emma Greenwood

\section{(D) References and links}

ORIGINAL RESEARCH PAPER Krtolica, A. et al. Senescent

fibroblasts promote epithelial cell growth and tumorigenesis: a link between cancer and aging. Proc. Natl Acad. Sci. USA 98, 12072-12077 (2001)

\section{WEB SITES}

Judith Campisi's lab:

http://www.lbl.gov/lifesciences/CMB/Campisi.html 